\title{
Evaluation of the Master's curriculum for elderly nursing: a qualitative study
}

\author{
This article was published in the following Dove Press journal: \\ Clinical Interventions in Aging \\ 26 September 2016 \\ Number of times this article has been viewed
}

\author{
Fatemeh Ghaffari ${ }^{1}$ \\ Nahid Dehghan-Nayeri² \\ Nasrin $\mathrm{Navabi}^{3}$ \\ Khatereh Seylani ${ }^{4}$ \\ 'Ramsar Nursing Care Research \\ Center, Babol University of Medical \\ Sciences, Mazandaran, ${ }^{2}$ Nursing and \\ Midwifery Care Research Center, \\ School of Nursing and Midwifery, \\ Tehran University of Medical Sciences, \\ Tehran, ${ }^{3}$ Nursing and Midwifery \\ School, Babol University of Medical \\ Sciences, Mazandaran, ${ }^{4}$ School of \\ Nursing and Midwifery, Tehran \\ University of Medical Sciences, Tehran, \\ Iran
}

\begin{abstract}
Background: Improving the quality of health care and rehabilitation for the elderly is one of the most important priorities of the health care system. Given the importance of evaluating the strengths and weaknesses of any program after its implementation, this study was conducted to identify the advantages and weaknesses of a geriatric nursing program at Tehran University of Medical Sciences.

Methods: This was a qualitative study, and the study population comprised students, graduates, and professors of geriatric nursing at the Master of Science level. Data were collected through face-to-face interviews and focus groups. Sixteen interviews were conducted. The interview guide was used as a research tool. Interviews continued until data saturation was reached. Conventional content analysis was used to analyze the data.

Results: Three main themes including "motivation to enter geriatric nursing", "lack of employment groundwork", and "lack of practical implementation of the curriculum" were the main findings of the study.

Conclusion: Efforts to restructure the administrative system and employment can deter geriatric nursing students from simply earning a degree and actually encourage them to learn the required content. Appraisal and improvement of education facilities for student recruitment can guarantee the practical implementation of the curriculum. Drafting policies to attract graduates in clinical environments, opening up employment opportunities, providing organizational positions for the recruitment of this group, as well as dedicating some wards for elderly special care and providing nursing care to elderly people only can increase students' motivation to learn and their hopes of good job prospects.
\end{abstract}

Keywords: Iran, geriatric nursing, content analysis, MS student

\section{Introduction}

Aging is a natural and universal process that continues throughout the life cycle; it does not signify disease and cannot be stopped or reversed. ${ }^{1}$ The world's population is rapidly aging. With an increase in the number of elderly persons, attention to the quality of life of this group is of utmost importance.

The most important achievement by humans in the 20th century has been an increase in life expectancy to $>66$ years. Therefore, after improvement of health, one of the major challenges for human societies in recent decades has been a significant increase in the number of elderly people. In future, $60 \%$ of people will live in developing countries that are not prepared to deal with the phenomenon of aging. ${ }^{2}$ According to the latest census, elderly people make up $8 \%$ of the Iranian population. Given the upward trend of the elderly population, it is anticipated that the number of elderly people will reach 10 million by the year 2021 . That will be $\sim 10 \%$ of the total population. ${ }^{3}$ 
A multidimensional approach involving numerous experts is ideal in dealing with the phenomenon of aging. This is because of the multidimensional nature of diseases and disabilities among the elderly, coupled with the significant burden posed by these conditions, a high prevalence of acute and chronic diseases, and additional problems in communication with and the treatment of this group. Although the aging of a population is a positive result of development, lack of preparation in dealing with the situation could result in negative consequences. ${ }^{4}$ However, evidence has shown that, despite an increase in the physical and mental health needs of elderly people, there is a significant shortage of geriatric nurses. ${ }^{5}$ Moreover, geriatric nurses do not have the necessary capabilities to diagnose elderly people's problems; they are also ill-prepared in dealing with the challenges associated with providing care to elderly people. ${ }^{6}$ Society's attitude toward aging has changed and, as a result, has highlighted a need for professional nurses with a unique body of knowledge and skills in caring for elderly people. ${ }^{7}$

The main philosophy of preparing nurses for working in the field of aging is provision of support to elderly clients in overcoming disability, improving their efficacy, empowering them, and helping them develop self-care skills. The postgraduate curriculum in this area should be more clinical and competency based. ${ }^{8}$

Since special needs influence conditions, traditions, and the dominant culture in any aging society, graduates should have enough knowledge and skills in providing care to elderly people, from prevention to rehabilitation. They should also be able to determine the special nursing needs of elderly people through the application of human communication principles to improve health services. ${ }^{9}$ Therefore, the curriculum will require innovation, evidence-based performance, and the setting of standards for geriatric nursing. Given the importance of geriatric nursing and the lack of expertise that this group has, a geriatric nursing curriculum at the Master's level was developed in Iran. The curriculum was implemented in 2010. Students take up this major through an entrance exam. Some students enter this level of study immediately after a Bachelor's degree; some acquire caring experience in various wards. Since the geriatric nursing curriculum was planned in response to the shortage of skilled nurses, its aim should be to equip students with a set of knowledge, attitudes, and skills needed to perform in the geriatric nursing profession. ${ }^{10}$ Therefore, it is emphasized that the curriculum should aim to train qualified nurses. ${ }^{11}$

Based on the abovementioned information, it is necessary to ensure that graduates are sufficiently qualified.
To enable provision of high-quality instruction, curricula must be revised regularly. ${ }^{12}$ One of the vital aspects in the evaluation of a curriculum is the identification of students' and faculty members' experiences. Since profound understanding of the challenges of this program cannot be achieved through a quantitative approach, the researchers sought to use qualitative methods to achieve this aim.

\section{Methods}

Based on the aim of this qualitative study, geriatric nursing students, graduates, and faculty members of Tehran University of Medical Sciences participated in the study. Purposive sampling was used for data collection. The sample consisted of 16 participants (13 students or graduates, and three faculty members of geriatric nursing department). Sixteen face-toface interviews were conducted. Two focus groups were conducted with three students, with the aim of obtaining data saturation. Two researchers were present at the focus groups. One of the researchers moderated the focus group, and the other noted the key points raised by the group.

At first, students and graduates were asked to express their experiences passing Master of Science (MS) level, and barriers and facilitators of the program's implementation. Interviews began with semi-structured questions, such as "How did you perceive studying in this field? "How did it prepare you as a geriatric nurse specialist?; What were the obstacles and facilitating factors in the achievement of the program's objectives?" Then, participants were probed in relation to their responses and based on the results of previous analyses. In addition, faculty members' views about the strengths and weaknesses of the curriculum were asked. Depending on the willingness of participants and the information that they provided, the interviews lasted between 35 minutes and 45 minutes, and focus groups 25 minutes and 35 minutes. Interviews were recorded through a digital mp3 recorder. Interviews were conducted in the researcher's office, in the School of Nursing, or where the participants felt comfortable. Sampling continued until the data were saturated and repetitive. Data collection began in 2014 and continued until 2015. The interviews were analyzed through Graneheim and Lundman's ${ }^{13}$ approach. Thus, all of the interviews were transcribed immediately after recording. Thereafter, the transcripts were read several times to achieve an overall understanding of the participants' experiences. The data were coded in three phases. At first, the semantic units or initial codes were extracted. Then, the codes were compared and classified according to similarities and differences, and, finally, themes were identified. 


\section{Trustworthiness}

The four criteria recommended by Guba and Lincoln were used..$^{14}$ The researcher spent a long period of time in the field ( $\sim 9$ months) interacting with participants and gathering data. We used a combination of face-to-face interviews and focus groups to collect the data. The codes were reviewed and confirmed by participants and some reviewers with expertise in this area. Maximum variant sampling was used (age, sex, work experience, and the gap between graduating from bachelor degree [BS] to entry into MS level). To enable transferability to other contexts, the researcher recorded the situation in detail.

\section{Ethical considerations}

The ethics committee of Tehran University of Medical Sciences approved the study. The participants were informed about the objectives of the study. Written informed consent was obtained from participants for participation, permission to record the interview for transcription, and anonymous publication of results. The time and place of the interviews were determined with participants' agreement. We assured them of anonymity and the confidentiality of their personal information and all the documents. The participants could withdraw from the study or request for exclusion of their data.

\section{Results}

Table 1 shows the participants' demographic characteristics.

Data analysis yielded 380 codes. Three main themes including "incentives to enter geriatric nursing", "lack of employment groundwork", and "lack of practical implementation of the curriculum" were the main findings of the study (Table 2).

\section{Incentives to enter geriatric nursing}

This category includes "degree oriented", "getting organizational posts", and "good chance for entrance".

\section{Degree oriented}

From most participants' perspective, being degree oriented is one of the most important factors in the pursuit of a Master's degree in geriatric nursing. The influence of degree orientation on community culture and the degree's relationship with social and occupational aspects have led some nurses to choose geriatric nursing, regardless of their personal interests and the hope for good job prospects.

I wanted to have postgraduate qualification. I feel that it is better if I continue with this geriatric nursing major. This will satisfy me. [Code 8 , student]

One faculty member says,

When we see all this indifference to learning, I do not think anything, just they come to get a degree and go. [Code 14, faculty member]

\section{Promotion in organizational posts}

Promotion in organizational posts is another factor in the continuation of geriatric nursing education at the MS level. Many students work in hospitals while studying. Therefore, they do not allocate enough time toward learning and assignments. Most students' aim was to get promoted within

Table I Participants' personal data

\begin{tabular}{|c|c|c|c|c|c|c|}
\hline No & $\begin{array}{l}\text { Age } \\
\text { (years) }\end{array}$ & Sex & $\begin{array}{l}\text { Marital } \\
\text { status }\end{array}$ & $\begin{array}{l}\text { Time interval between } \\
\text { BS and MS (years) }\end{array}$ & Type of contract & $\begin{array}{l}\text { Job experience } \\
\text { (years) }\end{array}$ \\
\hline I & 30 & Female & Single & 2 & Temporary contracts & I \\
\hline 2 & 29 & Female & Single & 3 & Fixed-term contracts & 2 \\
\hline 3 & 36 & Female & Married & 13 & Fixed-term contracts & 10 \\
\hline 4 & 44 & Female & Married & 18 & Fixed-term contracts & 16 \\
\hline 5 & 29 & Female & Single & 4 & Temporary contracts & 3 \\
\hline 6 & 36 & Male & Single & 12 & Fixed-term contracts & 11 \\
\hline 7 & 34 & Male & Married & 10 & Fixed-term contracts & 9 \\
\hline 8 & 32 & Female & Married & 8 & Fixed-term contracts & 8 \\
\hline 9 & 32 & Female & Married & 13 & Fixed-term contracts & 12 \\
\hline 10 & 32 & Female & Married & 8 & Fixed-term contracts & 8 \\
\hline II & 33 & Male & Married & 10 & Fixed-term contracts & 10 \\
\hline 12 & 46 & Female & Married & 18 & Fixed-term contracts & 17 \\
\hline 13 & 45 & Female & Married & 19 & Fixed-term contracts & 18 \\
\hline 14 & 49 & Male & Married & 7 & Faculty & 24 \\
\hline 15 & 48 & Female & Married & 9 & Faculty & 19 \\
\hline 16 & 49 & Female & Married & 12 & Faculty & 22 \\
\hline
\end{tabular}

Abbreviations: BS, Bachelor of Science; MS, Master of Science. 
Table 2 Categories and subcategories

\begin{tabular}{|c|c|}
\hline Categories & Subcategories \\
\hline $\begin{array}{l}\text { Incentives to enter } \\
\text { geriatric nursing }\end{array}$ & $\begin{array}{l}\text { Degree oriented } \\
\text { Promotion in organizational posts } \\
\text { Good chance for entrance }\end{array}$ \\
\hline $\begin{array}{l}\text { Lack of employment } \\
\text { groundwork } \\
\text { (no entry contexts) }\end{array}$ & $\begin{array}{l}\text { Specialization with a gray outlook } \\
\text { Goal setting without a comprehensive plan } \\
\text { The lack of cultural infrastructure }\end{array}$ \\
\hline $\begin{array}{l}\text { Lack of practical } \\
\text { implementation of } \\
\text { the curriculum }\end{array}$ & $\begin{array}{l}\text { The vicious cycle of BS lessons } \\
\text { Inefficiency of internships } \\
\text { Lack of guidance with student-centered tag/excuse } \\
\text { Ignoring cultural values in the curriculum }\end{array}$ \\
\hline
\end{tabular}

Abbreviation: BS, Bachelor of Science.

the organization after graduation. The aim of this group of nurses for pursuing a Master's degree was to be released from hospital work and to care directly for patients, taking up managerial positions and establishing care centers for elderly people.

I was tired and cannot stand all of this shift work, caring for 27 or 28 patients; then, answer all the questions of companion sand visitors. I wanted to be a supervisor. Then, I would not have to deal with patients and their companions. You will be a head nurse or a supervisor if you get an MS degree. [Code 9, student]

They want to continue their education because they want to get a position. It is the students' main aim. They are not interested in learning more and studying. [Code 14, faculty member]

\section{Good chance for entrance}

Another factor in the choice and entry into geriatric nursing, based on participants' views, was being new and being more capable of entry into this major, as compared to others.

Several times, I took part in the Master's entrance exam, but I was not accepted. This time, I chose geriatric nursing. It did not require a high ranking, in comparison with other nursing majors, and I was accepted. I was not interested in it. I just wanted to get my Master's degree. [Code 10, student]

\section{Lack of employment groundwork}

This main category includes "specialization with a gray outlook", "goal setting without a plan", and "the lack of cultural infrastructure".

\section{Specialization with a gray outlook}

Specialization is one of the goals of geriatric nursing education. Although some participants described specialization as enhancing the ability to solve problems associated with aging, and a change in nurses' attitudes toward elderly people and their special needs, they believed that there is insufficient groundwork to attract graduates into this field. Graduation in geriatric nursing is associated with some expectations, such as providing special care to elderly people, getting promotion in an organizational position, and having a change in one's social identity. However, these expectations are often not met. Therefore, job dissatisfaction, disappointment, and despair arise.

I am afraid of dedicating a few years towards studying, and then ultimately not reaping any outcomes and staying jobless. Now, I see that my friend is in the same ward that she was previously in; she cannot work, based on her expertise.

[Code 2, new graduate]

The interesting point in the finding is that most of these students do not feel a change in their position, as compared to before. Moreover, they do not apply their expertise in clinical practice. The absence of elderly wards in hospitals is an important factor. This creates role ambiguity, frustration, and a sense of futility of the education received at the MS level; these have been referred to as comprising a gray outlook.

Our hospital was not designed for elderly people. Most elderly people are hospitalized in different wards, among patients of varying ages, with different needs. This leads to the neglect of elderly people's needs and lack of provision of special elderly care; in such conditions, my expertise will not be applied. [Code 3, student]

You cannot say, "I studied geriatric nursing and I have more experience; so, I should work with elderly people, according to my specialty." There is no difference between MS and a Bachelor's. [Code 2, graduate]

Although the fact that the major is relatively new is one of the motivating factors in its being chosen, a lack of an organizational position for geriatric nursing graduates in hospitals, daily care provision to elderly people, old-age homes, and consulting centers frustrated them from enjoying a good job prospect in future. Most of the participants believed that geriatric nursing graduates can establish service centers such as nursing homes for elderly people, but physicians are leading in the establishment of these. As a result, this group of nurses has few opportunities in this field. Lack of career prospects is associated with a suppression of motivation for learning and continuing education, and with a sense of uncertainty.

They should not have established this major because there is no provision for that. They just wanted to add a major to 
nursing and accepted students. They do not know how to and where to recruit them. [Code 2, graduate]

The lack of a suitable working field is career threatening. Although most MS credits in geriatric nursing are related to gerontology and care for elderly patients, the lack of fieldwork limits job opportunities. They sometimes even encounter rejection in their application for work in general or critical units.

When I went to private hospitals for overtime work, they said, "We do not need your MS degree. If you want to work here, we will accept your BS." I was surprised as to why they established the curriculum when there is no labor market and we are required to work with a BS certificate. So, what is the point of it! [Code, 5]

\section{Goal setting without a comprehensive plan}

The purpose of training geriatric nurses is to impart skills in caring for elderly people. However, planning is often associated with many problems in achieving this goal and is sometimes not achieved.

Inconsistencies between lecturers of a given course, lack of control over the results of the internship, overlapping lessons, and the allocation of insufficient time toward some lessons are some of the problems associated with inaccurate planning, from participants' view. There are few opportunities to learn because of the remoteness or nonexistence of private centers.

We should go from Tehran to Kahrizak nursing home in Karaj and we had to travel for about two hours, for a one-day visit. We would like to compare this center with a private center, but often, private centers do not accept us.

[Code 4, student]

The educational management of the schools has resulted in aging specialists of other centers not being invited for teaching. Therefore, nonexperts gave lessons.

We have specialists who have been taught gerontology in Canada, or sociologists who have worked for years with elderly people, but they were not appointed to teach courses. [Code 13, student]

The provision of uncoordinated lessons by multiple lecturers was also problematic. This resulted in the repetition of some parts of lessons and others were omitted, and thus, reducing the quality of learning.

The greatest problem that I have encountered at this level was the recruitment of multiple lecturers or professors in a lesson. They repeated some parts and were uncoordinated, and it caused problems and we could not learn much. [Code 4, student]

Teaching by $\mathrm{PhD}$ graduates in nursing who had not passed gerontology courses or geriatric nursing was one of the problems with planning.

A number of lecturers did not have teaching or clinical experience; therefore, they were an important factor in the confusion experienced by students, especially in the internships.

We had a professor who had a PhD in nursing, but not in geriatric nursing; in addition, his thesis was not related to elderly people. He was good at teaching research or theories, but not in geriatric[s]. [Code 13]

We had two teachers in the lessons on structures.... They did not know how we should assess an elderly nursing home and extract its problems. They did not guide us well. Then, we wrote an assignment and did not get a good mark. [Code 11, student]

Delays in coordination with centers where students should do their internships were a major problem in educational planning. As a result, the centers did not accept students, who were confused and ended up losing time. Further, the process of elderly care provision was not comprehensive because of the lack of internship hours. They lost the opportunity to provide care as a case method, since the internships were interrupted. In most cases, nursing interventions and the evaluation of the nursing process were eliminated.

We did not provide a nursing intervention ... because internships were discontinued, and the next time we went there, the patient had been discharged or they had changed our wards. [Code 4, student]

Another important problem was the lack of time for lessons and the integration of lessons, such as statistics and research, and a decrease in effective instruction time for students. Classes were also being crammed into 2-3 days a week, with students becoming bored, and subsequently there was a decrease in the quality of their learning.

Most of us work in hospitals and travel from different cities to the university..., and when we arrive, we are tired. Classes are boring and intensive. Learning does not actually happen, and we do not have the opportunity to study and review lessons. [Code 9, student]

Most participants believed that students' appointment as assistant teachers for the theoretical aspects and the internship 
would increase their confidence and that they would try to learn more and commit to do their homework perfectly, as a result.

If we involve students and they present more hours in the university, and can teach under graduate students, it will be very helpful for their learning. [Faculty member, lecturer]

\section{Lack of cultural infrastructure}

Community culture and awareness of elderly people's rights and their needs for special care, as well as the importance of geriatric nursing as a profession, can positively influence the treatment and acceptance of geriatric nursing students and increase their motivation.

However, due to the lack of cultural infrastructure, most students are criticized for continuing education in geriatric nursing by the public and colleagues.

When I present at the ward for a shift, my colleagues ask, "what is this major that you chose! What do you get [from it]? Where do you want to be employed? Those are elderly people; so, what can you do for them, and is it useful?" [Code 10, student]

\section{Lack of practical implementation of the curriculum}

This main category includes "the vicious cycle of BS lessons", "inefficiency of internships", "lack of guidance with student-oriented tags", and "ignoring cultural values in the curriculum".

\section{Vicious cycle of BS lessons}

Participants mentioned that there was actually no difference between Bachelor's and Master's level courses, and that some of them were parallel, thereby resulting in a waste of time and energy and posing a threat to their future careers. Although it is necessary to provide topics with a focus on elderly persons, so as to change students' attitudes, in reality, this is often not achieved.

Lessons were taught in very general terms. In fact, they were the same as our BS lessons. We were taught what we studied in BS. They provide a little instruction about elderly people and it was not enough. [Code 2, graduate]

The study gap between BS and MS levels for some of the students has been more than 5 years, and this has led to educators assigning more time and energy toward covering concepts related to $\mathrm{BS}$. That is not consistent with the time devoted to study units. The rest of the students did not have any work experience because they were accepted immediately after BS graduation and encountered some challenges when caring for elderly people.

Our students have been working for several years and were accepted into MS after a long study gap; so, they have forgotten what they previously studied. They work routinely in the workplace without any studying; they do not update their knowledge. We have to repeat BS lessons again. If we do not cover them, they cannot understand the rest of the lessons. [Faculty member, lecturer]

According to our participants, internships for some units, such as pharmacology and epidemiology, were not possible, due to the lack of expert professors and a shortage of fieldwork.

Some internships are impossible, such as those in pharmacology, because we do not have an expert professor and related fieldwork. We have to miss this unit and, instead, provide that as a theoretical lesson. [Faculty member, lecturer]

\section{Ineffective internship}

According to most participants, emphasizing the theoretical courses and dedicating considerable time to them mean that the practical units and internships do not lead to skills acquisition, to facilitate the professionalization of geriatric nurses.

We could not manage an elderly patient; for example, write a plan for them or do something for him/her and, what is the problem? ... We did a little nursing intervention and we were not taught often. [Code 3]

The data showed that the unavailability of suitable fields for internships, such as elderly nursing homes, daily care centers, and cultural centers, were some of the reasons for the omission of internships or practical units, leading to clinical and hospital internships being replaced with visits to these places.

We went to hospitals just for visits, not to do internships. While we should learn how to take care of elderly people, we should determine their needs and plan for caring. We could not achieve this goal with a few days of internships. [Code 3, student]

It (internship) was not enough for gaining clinical skills; we only completed some questionnaires, and the aim or outcome of this was not clear. [Code 2, graduate]

A shortage of qualified instructors is another reason for the reluctance of educational planners to provide practical and functional units to geriatric nursing students. Most of the 
instructors are lecturers or newly qualified $\mathrm{PhD}$ graduates in nursing, currently holding internships. This group does not have the ability to manage geriatric nursing internship units, due to lack of education in this field. In fact, the practical units change to theory.

The internship was almost consistent with the theories, to some extent, but the instructors were not skillful, and the internships were not integrated. Most had no experience who can handle internship effectively. [Codes 2 and 3]

Significant consequences of the lack of clinical skills were loss of employment opportunities in the educational and clinical centers. Participants were often concerned about what will happen in their future careers and the options that they would have, since education and skills acquisition can guarantee their professional future.

The internship should not be a repetition of theoretical courses. We should train students and should expect a student to be able to practice in hospitals under the supervision of his/her mentor. If they can gain skills, they will stand a better chance for employment in different clinical environments. [Code 4]

\section{Lack of guidance with student tags}

Lack of professional instructors, resources, and proper planning for geriatric nursing has led to the delegation of the teaching of most outlines to students, while instructors do not guide students in this area. Students' inability to manage the classroom and lack of mastery and control over content play a role in the ineffectiveness of the selected method of education. The use of speech only in teaching topics and teaching in an unclear manner and without sufficient capability are the outcomes of the incomplete use of the student-oriented method.

The topics that instructors delegate to us for studying are subjects that they are not knowledgeable about or could not find any resources for. It is interesting that, when we send our Power Point slides to them, they say that they are good, but when we present them, they are not satisfied. So, why did they not offer us their guidance? [Code 11, student]

\section{Ignoring cultural values in the curriculum}

Cultural differences play a major role in education. However, there is an important gap in the curriculum. The provision of culture-based care can increase collaboration and the acceptance of self-care by elderly people. It can also increase active participation by the family.
In Islamic and Iranian culture, keeping elderly people at home is an important value, while it does not hold much value in industrial societies. It is a very important point that planners attend to this aspect. [Code 15 , faculty member]

\section{Discussion}

Three main themes, including "incentives to enter geriatric nursing", "lack of employment groundwork", and "lack of practical implementation of the curriculum", were the main findings of the study.

The results showed that the motivation for taking up the major included the willingness to obtain a formal certificate without qualification, promotion in the organization, and a high chance of acceptance in the entrance exam. These incentives are unlike the acquisition of skills and knowledge in the graduate courses and result in students memorizing series of content in the study materials. Therefore, they are left without the necessary skills in the labor market. The relationship between the certificate with dignity and social prestige and job status means that the certificate is a critical factor in one's social life. However, it is necessary to have the knowledge and expertise to replace being degree oriented. This is a process that requires the efforts of experts. A positive attitude toward the phenomenon of aging and the special needs of elderly people requires strong motivation and professional commitment; these are not consistent with being degree oriented. ${ }^{15}$ Moreover, considering this field as one promoting organizational positions leads to nurses not exerting effort in obtaining knowledge of geriatric nursing and not changing their attitude toward nursing care. As a result, this severely affects the quality of nursing care provided. ${ }^{16}$

The lack of employment groundwork for graduates was another important category in the findings. "Specialization with a gray outlook" was one of the subcategories. Although proficiency and the quality of care are the main goals of geriatric nursing, geriatric nurses are not recruited for special care for elderly people, due to the lack of employment groundwork in the clinical context and in communities. However, elderly people are anticipating high-quality nursing care that is associated with considerate and positive attitudes toward the elderly and their needs.

In expressing satisfaction with the quality of care, in a study by Raftopoulos, ${ }^{17}$ patients made a special mention of nursing care, including respect for the patient as a human being, specific technical skills for elderly people, a therapeutic touch, and empathy.

Bridges et $\mathrm{al}^{18}$ found that the main requests of elderly people to medical staff are good communication, respect for 
their identity, and their participation in decision making. The achievement of this goal is facilitated only through specialization and attention to the specific needs of elderly people.

A gray outlook that indicated a vague future for one's career was an important finding. Having a clear career future is one of the motivating factors for learning and trying to gain new skills and knowledge.

One's attainment of success and abilities should be accompanied by appropriate recognition of one's status. However, elderly nursing graduates experienced this to a considerably lesser extent. Their expertise made it difficult for them to get employed, as Iranian hospitals do not offer specialized services for elderly people. In these hospitals, most elderly people are hospitalized in the general wards and nursing care is provided by nurses with Bachelor's degrees. In this situation, these expert nurses cannot achieve a suitable job status and an organizational position. As the most important members of the health team, nurses play a key role in providing high-quality care to elderly people. ${ }^{19}$ Conditions such as an increase in the number of elderly patients referred to hospitals and a serious shortage of qualified nurses result in great challenges in the care of elderly people. ${ }^{16}$ The use of nonexpert nurses to provide care to elderly people also decreases the quality of care provided, which is bound to increase the recovery period and other side effects. ${ }^{20}$

"Goal setting without a plan", which results in graduates being unable to find appropriate jobs, was another important finding. The lack of expert instructors in the geriatric field and an inefficient program including the omission of a series of theoretical and practical lessons, integration of some lessons and a decrease in their duration, an inappropriate order of and lack of coordination between the courses, a reduced internship period, and the delegation of teaching to unqualified students without any guidance, are the factors that challenge students' learning.

Instructors' knowledge and skills in theory and practice play a vital role in the promotion of students' practical skills and attitudes. Nursing instructors are responsible for the development of teaching skills that are appropriate for the clinical setting, because they not only teach but also serve as models for nursing care. ${ }^{21}$

Instructors believe that being model oriented in clinical settings is the most effective way of transferring professional experience and attitudes to students. ${ }^{22}$ Moreover, the clinical environment creates opportunities for students to see patterns, practice, and improve skills and abilities in problem solving. ${ }^{23}$ They will learn the positive attitude required in care provision to elderly people from the instructors. ${ }^{16}$
Lack of practical implementation of the curriculum was another important category. Students' circumstances, lack of access to geriatric nursing resources, some instructors' inability to use English texts, and their lack of experience or expertise in special geriatric nursing courses have resulted in the repetition of BSc courses at the MS level. This does not result in students' learning and also suppresses their motivation to learn.

Cognitive development and a positive attitude by students toward elderly people are consequences of specific instruction in geriatric nursing. However, achieving this goal is difficult, due to duplicate courses. Rahimi and $\mathrm{Ahmadi}^{24}$ have found that nursing instructors, particularly those in specialized fields, often lack the necessary expertise to transfer their knowledge and experiences to learners. Loscocco ${ }^{25}$ study showed that students are dissatisfied as a result of some components, such as a lack of instructors' knowledge, coherence between subjects, and inefficiencies and gaps in some subjects. In addition, a study by Routasalo et al entitled, "Perceptions and experiences of students about a geriatric nursing education program", explored four themes, including instructors' skills, a combination of quality and safety in the curriculum, teaching through use of real educational strategies, and professional growth opportunities. ${ }^{26}$

The results of this study indicate that clinical centers are limited for internships, and these courses do not have varying learning outcomes. The lack of application of subjects learned; ineffective implementation of some nursing care, such as education and a good relationship with elderly people and their families; and the failure to assess the status of elderly people and to identify and solve their health problems were among the weaknesses of internship courses. Important outcomes of this situation were frustration, loss of motivation, and forgetting what has been learned. The main mission of nursing education is the training of competent nurses who have the knowledge, attitudes, and skills necessary to improve the health of communities. ${ }^{27}$ Clinical education plays an important role in the achievement of this aim. Nursing success substantially depends on the effectiveness of clinical experiences. ${ }^{28}$ The results of a study by Bednash et $\mathrm{al}^{29}$ about the "effects of curriculum on learning" showed that $85 \%$ of students regarded their clinical experiences of taking care of elderly individuals as incomplete.

Incorrect application of the student-oriented approach as a teaching method of choice in geriatric nursing education is another challenge that leads to a decrease in the quality of learning. According to most participants' view, this method does not facilitate the learning process and, because of students' lack of experience in teaching, the understanding of subjects is not associated with practical examples. Therefore, students are not driven to use theory in practice. ${ }^{30}$ 


\section{Conclusion}

The result of the study showed that the incentives to enter the field of geriatric nursing include matters other than the acquisition of knowledge, skills, and specialization. Efforts to restructure the administrative and employment systems can modify the tendency toward being degree oriented and emphasis on promotion, substituting this with learning and increased expertise.

The provision of job opportunities by policymakers, so as to attract graduates of geriatric nursing, which is now a gray outlook, coupled with specialization by wards to specifically for elderly people, can guarantee the future of geriatric work. Periodic audits of education centers, processes, and curriculum outcomes can improve the quality of the training provided to geriatric nurses.

The lack of geriatric nursing faculty and internship centers modified practical units into theory. In addition, due to the lack of specialist instructors, educational quality has decreased. Authorities and curriculum planners can invite expert professors to improve the quality of the education imparted. They can also develop workshops and in-service education for faculty members. In addition, periodic quantitative and qualitative evaluation of the curriculum is suggested for obtaining the necessary feedback that will lead to curriculum improvement.

\section{Acknowledgments}

The study protocol of this research was approved by Tehran University of Medical Sciences, Nursing and Midwifery Care Research Center, No 22245. The authors appreciate all the participants who shared their experiences with us.

\section{Author contributions}

Dr Fatemeh Ghaffari participated in data analysis and writing of the manuscript. Dr Nahid Dehghan-Nayeri participated in writing both the manuscript and the proposal. Ms Nasrin Navabi and Dr Khatereh Seylani participated in data collection. All authors contributed toward data analysis, drafting and critically revising the paper and agree to be accountable for all aspects of the work.

\section{Disclosure}

The authors report no conflicts of interest in this work.

\section{References}

1. Tabloski PA. Gerontological Nursing. Upper Saddle River: Pearson Prentice Hall; 2006.

2. Ebersole P, Touhy TA. Geriatric Nursing: Growth of a Specialty. New York: Springer Publishing Company; 2006.
3. Azizi A, Abedi H. Study the status of daily activities of living in elderly with Alzheimer. Int J Basic Sci Appl Res. 2014;3(SP):131-134.

4. Stoesz D. Age concerns: innovation through care management. J Aging Soc Policy. 2002;14(3-4):245-260.

5. Wright J, McCormack B. Practice development: individualised care Nurs Stand. 2001;15(36):37.

6. Kovner CT, Mezey M, Harrington C. Who cares for older adults? Workforce implications of an aging society. Health Aff. 2002;21(5):78-89.

7. Eliopoulos C. Gerontological Nursing. Philadelphia: Lippincott Williams \& Wilkins; 2013.

8. Stone JT, Wyman JF, Salisbury SA. Clinical Gerontological Nursing. A Guide to Advanced Practice. Philadelphia: Saunders; 1999.

9. Waite LJ. Aging, Health, and Public Policy: Demographic and Economic Perspectives. New York: Population Council; 2004.

10. Resnick B. Group reflections on building academic geriatric nursing capacity. Geriatr Nurs. 2012;33(2):83-85.

11. Pierre JS, Twibell R. Developing nurses' geriatric expertise through the geriatric resource nurse model. Geriatr Nurs. 2012;33(2):140-149.

12. Spruill IJ. Curriculum development in nursing: process and innovation. ABNF J. 2006;17(4):152.

13. Graneheim UH, Lundman B. Qualitative content analysis in nursing research: concepts, procedures and measures to achieve trustworthiness. Nurse Educ Today. 2004;24(2):105-112.

14. Guba EG, Lincoln YS. Competing paradigms in qualitative research. Handbook of Qualitative Research. 1994;2(163-194):105-117.

15. Lovell M. Caring for the elderly: changing perceptions and attitudes. J Vasc Nurs. 2006;24(1):22-26.

16. Purfarzad Z, Ghorbani M. Teachers and student nurses' attitudes caring for older adults in Arak, 2012. J Clin Nurs Midwifery. 2014;3(2): 46-56

17. Raftopoulos V. Assessment of users' expectations, perceived quality and satisfaction with primary care in Greece. Int J. 2010;3(3):110.

18. Bridges J, Flatley M, Meyer J. Older people's and relatives' experiences in acute care settings: systematic review and synthesis of qualitative studies. Int J Nurs Stud. 2010;47(1):89-107.

19. Hweidi IM, Al-Obeisat SM. Jordanian nursing students' attitudes toward the elderly. Nurse Educ Today. 2006;26(1):23-30.

20. Kennedy-Malone L, Penrod J, Kohlenberg EM, et al. Integrating gerontology competencies into graduate nursing programs. J Prof Nurs. 2006;22(2):123-128.

21. Maag MM, Buccheri R, Capella E, Jennings DL. A conceptual framework for a clinical nurse leader program. J Prof Nurs. 2006;22(6):367-372.

22. Hossein KM, Fatemeh D, Fatemeh OS, Katri VJ, Tahereh B. Teaching style in clinical nursing education: a qualitative study of Iranian nursing teachers' experiences. Nurse Educ Pract. 2010;10(1):8-12.

23. Twentyman M, Eaton E, Henderson A. Enhancing support for nursing students in the clinical setting. Nurs Times. 2006;102(14):35.

24. Rahimi A, Ahmadi F. The obstacles and improving strategies of clinical education from the viewpoints of clinical instructors in Tehran's Nursing Schools. Iran J Med Educ. 2005;5(2):73-80.

25. Loscocco K. Teaching Graduate Students to Teach Sociology. Report of the Ad Hoc Graduate Education Committee. Washington, DC: American Sociological Association; 1996.

26. Routasalo P, Wagner L, Virtanen H. Registered Nurses' perceptions of geriatric rehabilitation nursing in three Scandinavian countries. Scandinavian Journal of Caring Sciences. 2004;18(2):220-228.

27. Li Y-S, Chen P-S, Tsai S-J. A comparison of the learning styles among different nursing programs in Taiwan: implications for nursing education. Nurse Educ Today. 2008;28(1):70-76.

28. Peyrovi H, Yadavar-Nikravesh M, Oskouie SF, Berterö C. Iranian student nurses' experiences of clinical placement. Int Nurs Rev. 2005; 52(2):134-141.

29. Bednash G, Mezey M, Tagliareni E. The Hartford Geriatric Nursing Initiative experience in geriatric nursing education: looking back, looking forward. Nurs Outlook. 2011;59(4):228-235.

30. Carlson ML, Ivnik MA, Dierkhising RA, O’Byrne MM, Vickers KS. A learning needs assessment of patients with COPD. Medsurg Nurs. 2006;15(4):204 


\section{Publish your work in this journal}

Clinical Interventions in Aging is an international, peer-reviewed journal focusing on evidence-based reports on the value or lack thereof of treatments intended to prevent or delay the onset of maladaptive correlates of aging in human beings. This journal is indexed on PubMed Central, MedLine,

CAS, Scopus and the Elsevier Bibliographic databases. The manuscript management system is completely online and includes a very quick and fair peer-review system, which is all easy to use. Visit http://www.dovepress. com/testimonials.php to read real quotes from published authors. 